\title{
Increased PD-L1 and T-cell infiltration in the presence of HLA class I expression in metastatic high-grade osteosarcoma: a rationale for T-cell-based immunotherapy
}

\author{
Yayan T. Sundara $^{1} \cdot$ Marie Kostine $^{1,2} \cdot$ Arjen H. G. Cleven $^{1} \cdot$ Judith V. M. G. Bovée $^{1} \cdot$ \\ Marco W. Schilham ${ }^{3}$ Anne-Marie Cleton-Jansen ${ }^{1}$ (1)
}

Received: 16 August 2016 / Accepted: 4 November 2016 / Published online: 16 November 2016

(c) The Author(s) 2016. This article is published with open access at Springerlink.com

\begin{abstract}
Introduction Immunotherapy may be an excellent choice for treating osteosarcoma given its exceptionally high genomic instability, potentially generating neoantigens. In this study, we aim to investigate the HLA class I expression, PD-L1 and tumour-infiltrating lymphocytes in primary osteosarcomas and relapses/metastases, as well as their changes during disease progression.

Materials and methods Tumour samples from multiple stages of the disease (pretreatment biopsies, surgical resections of primary osteosarcomas, relapses and metastases) were collected and stained for HLA-A (HCA2), HLA-B/C (HC10), $\beta 2$-microglobulin and PD-L1 using immunohistochemistry on whole sections. Density and type of T-cell infiltrate were characterised by a triple immunofluorescent staining CD3-CD8-FOXP3.

Results Overall, 85 formalin-fixed, paraffin-embedded blocks from 25 osteosarcoma patients were included.
\end{abstract}

Yayan T. Sundara and Marie Kostine have contributed equally to this work.

Electronic supplementary material The online version of this article (doi:10.1007/s00262-016-1925-3) contains supplementary material, which is available to authorized users.

Anne-Marie Cleton-Jansen

a.m.cleton-jansen@lumc.nl

1 Department of Pathology, Leiden University Medical Center, P.O. Box 9600, 2300 RC Leiden, The Netherlands

2 Department of Rheumatology, Hôpital Pellegrin, Centre Hospitalier Universitaire de Bordeaux, Bordeaux, France

3 Department of Pediatrics, Leiden University Medical Center, P.O. Box 9600, 2300 RC Leiden, The Netherlands
HLA class I expression was detected in $94 \%$ of osteosarcomas (strongly positive in $56 \%$, heterogeneous in $38 \%$ ) and negative or weakly positive in $6 \%$, without differences between the stages of the disease. HLA-A expression was more frequently negative than HLA-B/C. Tumour-infiltrating lymphocytes were highly heterogeneous and mainly observed in tumour areas with expression of HLA class I. Density of T cells was significantly higher in metastases than in primary tumours and local relapses $(p=0.0003)$. Positive PD-L1 expression was found in $13 \%$ of primary tumours, $25 \%$ of relapses and $48 \%$ of metastases and correlated with a high T-cell infiltrate $(p=0.002)$.

Conclusion An increased number of tumour-infiltrating $\mathrm{T}$ cells and PD-L1 expression in metastases compared with primary tumours, suggesting accessibility for $\mathrm{T}$ cells, could imply that osteosarcoma patients with metastatic disease may benefit from T-cell-based immunotherapy.

Keywords Osteosarcoma $\cdot$ HLA class I - Tumourinfiltrating lymphocytes $\cdot$ PD-L1 $\cdot$ Immunotherapy

$\begin{array}{ll}\text { Abbreviations } \\ \text { CD } & \text { Cluster of differentiation } \\ \text { CTLA } & \text { Cytolytic T lymphocyte-associated antigen } \\ \text { DAB } & 3,3^{\prime} \text {-diaminobenzidine } \\ \text { DAPI } & 4^{\prime}, 6^{\prime} \text {-diamidino-2-phenylindole } \\ \text { EDTA } & \text { Ethylenediaminetetraacetic acid } \\ \text { FOXP3 } & \text { Forkhead box p3 } \\ \text { HLA } & \text { Human histocompatibility leucocyte antigen } \\ \text { HRP } & \text { Horseradish peroxidase } \\ \text { mRNA } & \text { Messenger RNA } \\ \text { NK cell } & \text { Natural killer cell } \\ \text { PD-1 } & \text { Programmed death 1 receptor } \\ \text { PD-L1 } & \text { Programmed death ligand 1 }\end{array}$




\section{Introduction}

Osteosarcoma is the most common primary high-grade bone malignancy, primarily affecting children and adolescents [1]. It commonly arises in the metaphyseal plates of the long bones of the extremities (i.e. distal femur, proximal tibia), while tumours developing in the axial skeleton, pelvis or craniofacial bones tend to occur in older individuals. Osteosarcoma patients are treated with curative intent, consisting of surgery of the primary tumour and any resectable metastatic lesions, in addition to pre- and post-operative chemotherapy [2]. This multimodal therapeutic approach greatly improves the disease-free survival probability, from 10 to $20 \%$ with the surgery alone, to more than $60 \%$. However, survival for patients with relapsed or metastatic disease remains dismal and unchanged over the last three decades, as efforts in developing novel active agents have been generally disappointing [3, 4]. Osteosarcoma's genomic complexity is one of the major explanations for the lack of specific targetable mutations or molecular pathways. On the other hand, this genomic characteristic and the associated high mutational burden may generate specific tumour neoantigens, therefore providing potential targets for T-cell-based immunotherapies [5-7].

Historically, the first successful example of immunotherapy was in 1891 when William B. Coley injected a mixture of streptococcal bacteria into unresectable bone sarcomas, resulting in an immunological reaction and tumour regression $[8,9]$. Recent advances in cancer immunology have now revealed the importance of a spontaneous antitumour immune reaction to predict response to immunotherapy, mostly carried out by cytotoxic CD8-positive T cells [10]. Antigen presentation by surface HLA expression on tumour cells is an important prerequisite for antitumour immunity since loss or down-regulation of HLA molecules is a common mechanism deployed by tumour cells to escape immune surveillance [11]. Another immune escape mechanism is the dysregulation of immune checkpoint pathways such as PD-1/PD-L1 axis, which has been actively studied in epithelial malignancies [12]. So far, data regarding the immune microenvironment in osteosarcoma are rather limited. In order to evaluate the feasibility of T-cell-mediated immunotherapies, we assessed HLA class I expression, PD-L1 and T-cell infiltration, as well as their changes during osteosarcoma progression using immunohistochemistry.

\section{Materials and methods}

\section{Patient material}

Formalin-fixed, paraffin-embedded materials from osteosarcoma patients with a known stable $(n=7)$ or progressive disease $(n=18)$ diagnosed between 1998 and 2011 were retrieved from the archives of the Pathology Department of the Leiden University Medical Center (LUMC). Patients characteristics are described in Table 1. Overall, 85 tissue blocks from 25 patients were collected for this study, including pretreatment biopsies $(n=16)$ and surgical resections of primary tumours $(n=18)$, local relapses $(n=25)$ and metastases $(n=26)$. Material from multiple stages of the disease was available for 17 patients, and five patients had metastatic disease at diagnosis (Supplementary Table 1). Whole sections were used to assess HLA class I expression, PD-L1 and T-cell infiltrate in these tumours. Diagnoses were confirmed by an experienced bone and soft-tissue tumour pathologist (J.V.M.G. Bovée) according to the 2013 World Health Organization classification. All the specimens were handled in a coded fashion according to the Code for Proper Secondary Use of Human Tissue in the Netherlands of the Dutch Federation of Medical Scientific Societies.

\section{Immunohistochemistry and scoring}

Immunohistochemistry stainings for HLA class I (HCA2, HC10 and 32 -microglobulin) and PD-L1 were performed according to standard laboratory protocols, as described previously [13]. Briefly, 4- $\mu \mathrm{m}$ sections were deparaffinised with xylene and rehydrated in graded concentrations of ethanol. Endogenous peroxidase was blocked in $0.3 \% \mathrm{H}_{2} \mathrm{O}_{2}$ solution, and microwave antigen retrieval was performed in Citrate pH 6.0 (for HLA class I) or in Tris-EDTA pH 9.0 (for PD-L1). Subsequently, sections were incubated

Table 1 Patient characteristics

\begin{tabular}{lr}
\hline$n$ & 25 \\
\hline Median age (years) & $18(7-70)$ \\
Gender & \\
Female & $8(32 \%)$ \\
Male & $17(68 \%)$ \\
Pre-operative chemotherapy & \\
Yes & $20(80 \%)$ \\
No & $5(20 \%)$ \\
Histological response & \\
Good & $14(56 \%)$ \\
Poor & $6(24 \%)$ \\
Unknown & $5(20 \%)$ \\
Median follow-up (months) & $56(14-117)$ \\
Progressive disease & \\
No & $7(28 \%)$ \\
Yes & $18(72 \%)$ \\
Metastases at time of initial diagnosis & \\
No & $20(80 \%)$ \\
Yes & $5(20 \%)$ \\
\hline
\end{tabular}


overnight at $4{ }^{\circ} \mathrm{C}$ with the different primary antibodies, as detailed in Table 2. The next day, the staining was detected using poly-HRP and visualised with a DAB + substrate chromogen system. Slides were counterstained with haematoxylin, dehydrated and mounted with $\mathrm{CV}$ mount (Leica Microsystems). Tonsil normal tissue sections were used as positive controls, and primary antibodies were omitted for the negative controls. When loss of tissue occurred during the staining procedure, specimens were not included in the analysis.

Whole sections were scored independently by two observers (Y.T. Sundara and J.V.M.G. Bovée or A.H.G. Cleven). As semiquantitative scores were used, there was a high concordance between observers and in case of discrepancies, the slide was reviewed to reach a consensus. The expression of HLA-A (HCA2 staining), HLA-B/C (HC10 staining) and $\beta 2$-microglobulin was assessed semiquantitatively as negative/focal weak (tumour cells negative or focally and weakly positive with positive internal controls), heterogeneous (both negative and positive regions on the same slide) and positive (moderate or strong staining in the whole tumour), as used in a previous study [13]. Because the light chain $\beta 2$-microglobulin is an essential constant component of HLA class I molecules, the final HLA class I expression status was determined according to the positivity of at least one of the heavy chains stainings (either HCA2, HC10 or both), combined with the $\beta 2$-microglobulin positivity score. Moreover, as many tumours were found heterogeneous for the different HLA stainings, we also assessed the degree of colocalisation between $\beta 2$-microglobulin and HCA2/HC10. This HLA scoring is detailed in Fig. 1e. Positive PD-L1 expression was defined as $\geq 1 \%$ of tumour cells or immune cells showing a membranous staining of any intensity, according to published data [14]. Additionally, sections with heterogeneous expression of HLA class I were scanned using Panoramic MIDI scanner (3DHISTECH Ltd.) to assess colocalisation with T-cell infiltration.

\section{Immunofluorescent staining and scoring}

For the detection and the characterisation of tumour-infiltrating $\mathrm{T}$ cells, we used a triple immunofluorescent staining, as previously described [15]. After antigen retrieval using Tris-EDTA buffer $\mathrm{pH}$ 9.0, whole sections were incubated overnight at $4{ }^{\circ} \mathrm{C}$ with the combination of primary antibodies, as detailed in Table 2 . The following day, isotype-specific secondary antibodies labelled with Alexa fluorochromes (Life Technologies) were added during $1 \mathrm{~h}$ at room temperature and slides were mounted using Vectashield mounting medium containing DAPI (Vector Laboratories).

Stained sections were observed at $250 \times$ magnification using a confocal scanning microscope (Zeiss LSM 700), and four randomly selected images were processed with the ZEN software (version 2.1, Carl Zeiss). The numbers of $\mathrm{CD}^{+} \mathrm{CD}^{-}$(membranous red staining), $\mathrm{CD}^{+} \mathrm{CD}^{+}$ (membranous purple staining) and $\mathrm{CD}^{+}{ }^{+} \mathrm{CD} 8^{-} \mathrm{FOXP} 3^{+}$ (green nuclear staining) $\mathrm{T}$ cells were counted using the cell counter plug-in of the program ImageJ version 1.48, and the results of the four images were averaged.

\section{Statistical analysis}

All statistical analyses were performed using SPSS software version 23.0 (IBM Corporation, New York, USA), and graphs were constructed using GraphPad Prism software version 6 (La Jolla, California, USA). Correlations between immunohistochemical data and clinicopathological variables were analysed using Spearman's rank correlation coefficient and Mann-Whitney $U$ tests. For patients with material from different stages of the disease, paired $t$ tests were used. A binary logistic regression model was used for predicting T-cell infiltration based on HLA class I expression. Correlations between immune markers and patient survival were tested using Kaplan-Meier survival analysis. Survival curves with immune data from primary tumours

Table 2 Characteristics of the antibodies and reagents used for immunohistochemistry (IHC) and immunofluorescence (IF)

\begin{tabular}{|c|c|c|c|c|c|c|c|}
\hline Antigen & Antibody supplier & Clone/reference & Isotype & Antibody dilution & Secondary reagent & Reagent supplier & Reagent reference \\
\hline \multicolumn{8}{|l|}{ IHC } \\
\hline $\mathrm{HCA} 2$ & Nordic-Mubio & MUB2036P & Mouse IgG1 & $1 / 3200$ & BrightVision & Immunologic & DPVO-HRP \\
\hline HC10 & Nordic-Mubio & MUB2037P & Mouse IgG2a & $1 / 3200$ & BrightVision & Immunologic & DPVO-HRP \\
\hline$\beta 2 \mathrm{~m}$ & Dako & A0072 & Rabbit IgG & $1 / 1600$ & BrightVision & Immunologic & DPVO-HRP \\
\hline PD-L1 & Cell signaling & E1L3 N & Rabbit IgG & $1 / 400$ & BrightVision & Immunologic & DPVO-HRP \\
\hline \multicolumn{8}{|l|}{ IF } \\
\hline CD3 & Dako & A0452 & Rabbit IgG & $1 / 400$ & $\mathrm{G} \alpha \mathrm{R}$ IgG-A546 & Invitrogen & A11010 \\
\hline CD8 & Novocastra & 4B11 & Mouse IgG2b & $1 / 200$ & $\mathrm{G} \alpha \mathrm{M}$ IgG2b-A647 & Invitrogen & A21242 \\
\hline FoxP3 & Abcam & 236A/E7 & Mouse IgG1 & $1 / 100$ & $\mathrm{G} \alpha \mathrm{M}$ IgG1-A488 & Invitrogen & A21121 \\
\hline
\end{tabular}

$\alpha$ anti, $A$ Alexa Fluor labelled, $G$ goat, $M$ mouse 
a

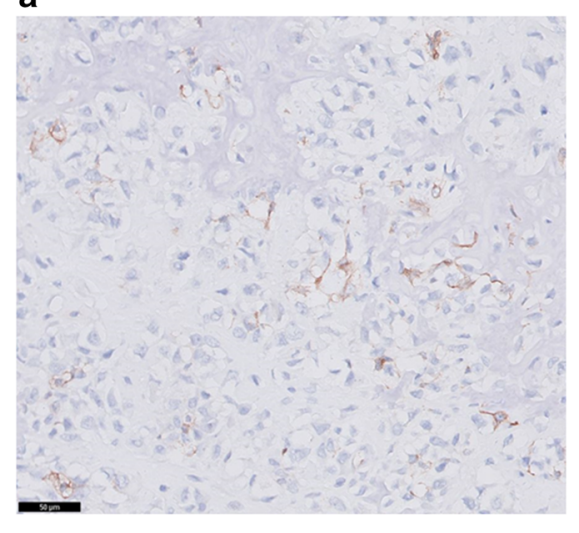

d

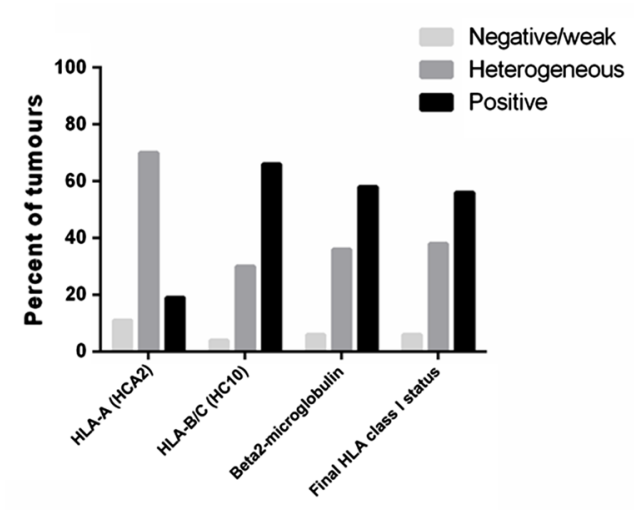

b

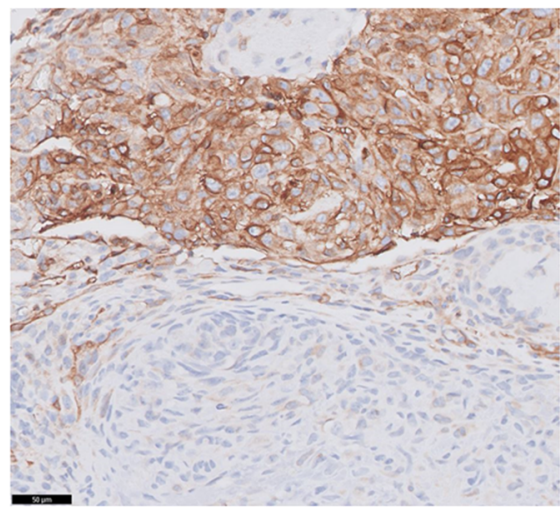

c

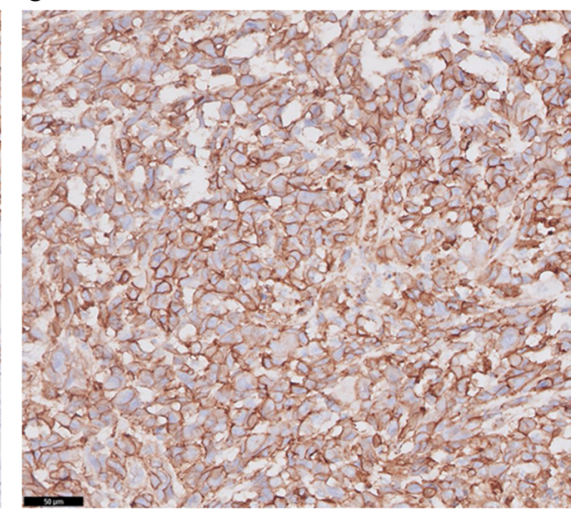

e

\begin{tabular}{|c|c|c|}
\hline$\beta 2 \mathrm{~m}$ & HCA2/HC10 & HLA clas s I scoring \\
\hline \multirow{4}{*}{ not defined } & not defined & \multirow{4}{*}{ not defined $(n=2)$} \\
\hline & negative & \\
\hline & heterogeneous & \\
\hline & negative & \\
\hline \multirow{4}{*}{ negative } & not defined & \multirow{4}{*}{ negative $(n=5)$} \\
\hline & negative & \\
\hline & heterogeneous & \\
\hline & positive & \\
\hline \multirow{4}{*}{ heterogeneous } & not defined & not defined $(n=0)$ \\
\hline & negative & negative $(n=0)$ \\
\hline & heterogeneous & \multirow{2}{*}{ heterogeneous $(n=28)^{*}$} \\
\hline & positive & \\
\hline \multirow{4}{*}{ positive } & not defined & not defined $(n=1)$ \\
\hline & negative & negative $(n=0)$ \\
\hline & heterogeneous & heterogeneous if colocalisation $<50 \%(n=2)$ \\
\hline & positive & positive ( $n=45$ ) \\
\hline
\end{tabular}

Fig. 1 Different HLA class I phenotypes in osteosarcoma. Representative staining patterns of HLA-A expression using immunohistochemistry (HCA2 antibody): negative/weak expression with endothelial cells as positive internal controls (a), heterogeneous expression with both negative and positive regions (b) and diffuse positive expression (c). Scale bars $50 \mu \mathrm{m}$. Negative and heteroge-

were generated based on biopsy specimens (16 patients), which reflect the natural host immune response and limit the impact of any neoadjuvant treatment on tumour microenvironment, or based on resection specimens when biopsy material was not available (six patients). Data from the first metastatic lesion were used for assessing overall survival from the time of diagnosis of metastatic disease (16 patients). $P$ values below 0.05 were considered statistically significant.

\section{Results}

\section{HLA-A is more frequently negative or heterogeneous than HLA-B/C in osteosarcoma}

HLA class I expression was determined on whole tumour sections by immunohistochemistry using the antibodies HCA2 (HLA-A), HC10 (HLA-B/C) and $\beta 2$-microglobulin. neous expression was observed more frequently for HLA-A compared to HLA-B/C (d). The final HLA class I expression status was determined according to the positivity of at least one of the heavy chains stainings (either HCA2, HC10 or both), dependent to the $\beta 2$-microglobulin positivity score (e)

Expression of HCA2 and HC10 was mostly membranous while expression of $\beta 2$-microglobulin was both membranous and cytoplasmic. Representative images of the different patterns of HLA class I expression (negative/ weak, heterogeneous and positive) are shown in Fig. 1a-c and Supplementary Figure 1. A selective lower HLA-A expression was more frequently observed than HLA-B/C, with $11 \%(n=9)$ of the tumours that did not or weakly expressed HLA-A and 70\% $(n=57)$ displayed a heterogeneous expression versus $4 \%(n=3)$ and $30 \%(n=24)$, respectively, for HLA-B/C (Fig. 1d and Supplementary Table 1). Overall, HLA class I expression was strongly positive in 56\% ( $n=45)$, heterogeneous in $38 \%(n=30)$, and negative or weakly positive in $6 \%(n=5)$ of 80 evaluated tumours, using the scoring described in Fig. 1e. No significant difference in HLA class I expression was observed between primary tumours, relapses and metastases ( $p=0.58)$, neither between pretreatment biopsies and surgical resections $(p=0.48)$. For the patients with metastases 
at diagnosis, the expression status of HLA class I in the primary tumour and associated synchronous metastasis was found similar while it mainly differed for the patients with metachronous metastases (Supplementary Table 2).

HLA class I expression was also evaluated on normal bone cells, when present. Osteocytes were mainly negative for both HLA-A and HLA-B/C, with a more variable expression of $\beta 2$-microglobulin. A positive but weak expression of all markers was often detected on osteoblasts (Supplementary Figure 2).

\section{Density of tumour-infiltrating lymphocytes is higher in metastatic lesions}

The presence and type of tumour-infiltrating $\mathrm{T}$ cells were determined by a triple immunofluorescent staining (CD3CD8-FOXP3) on whole osteosarcoma sections. Representative images are presented in Fig. 2a-c and Supplementary Figure 3. Density of $\mathrm{T}$ cells, defined as CD3 expressing cells, was significantly higher in metastatic lesions (mean $\pm \mathrm{SE}=75 \pm 13 \mathrm{CD}^{+}$cells) than in primary tumours $\left(19 \pm 6 \mathrm{CD}^{+}\right.$cells $)$and local relapses $(18 \pm 4$ $\mathrm{CD}^{+}$cells) $(p=0.0003)$, as shown in Fig. 2 d. In primary osteosarcomas, $46 \%$ of these tumour-infiltrating $\mathrm{T}$ cells were $\mathrm{CD}^{+} \mathrm{CD}^{+} \mathrm{T}$ cells, $52 \%$ in local relapses and $47 \%$ in metastases. $\mathrm{CD}^{+} \mathrm{CD}^{-}$cells (presumably composed of $\mathrm{CD}^{+}{ }^{+}$and $\gamma \delta \mathrm{T}$ cells) constituted 49 and $47 \%$ of the T-cell infiltrate in primary tumours and metastases, while this proportion was observed lower $(36 \%)$ but without statistical significance ( $p=0.73$ ) in local relapses (Fig. 2e and Supplementary Table 1). The local relapses displayed a higher proportion $(12 \%)$ of $\mathrm{CD}^{+} \mathrm{CD}^{-} \mathrm{FOXP}^{+} \mathrm{T}$ cells, compared to primary tumours $(4 \% ; p=0.15)$ and metastases $(6 \% ; p=0.024)$. We did not observe $\mathrm{CD} 3{ }^{+} \mathrm{CD} 8^{+} \mathrm{FOXP} 3^{+}$ cells.

\section{Correlation between HLA class I expression and tumour-infiltrating $\mathrm{T}$ cells}

We attempted to predict $\mathrm{T}$-cell infiltration based on the expression of HLA-A, HLA-B/C and $\beta 2$-microglobulin as a single independent variable or combined inter-dependent variables, using a binary logistic regression model. We found that the expression status of $\beta 2$-microglobulin together with the expression of HLA-B/C was a significant predictor for the total T-cell count $(p=0.04)$, but not if a

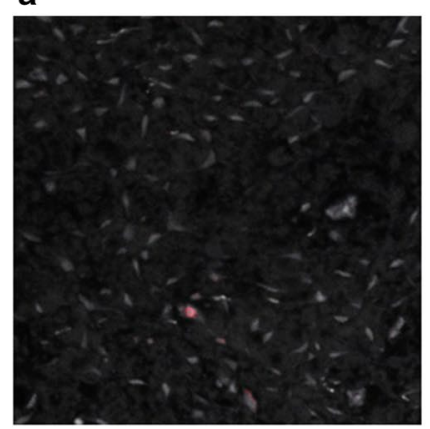

\section{b}

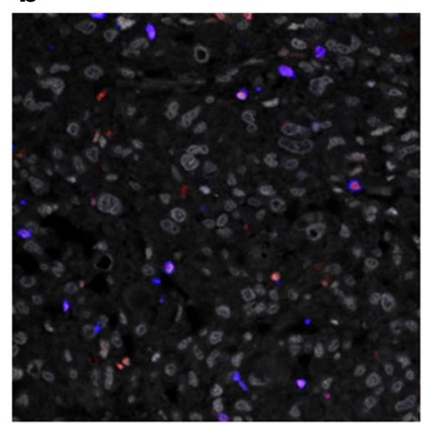

C

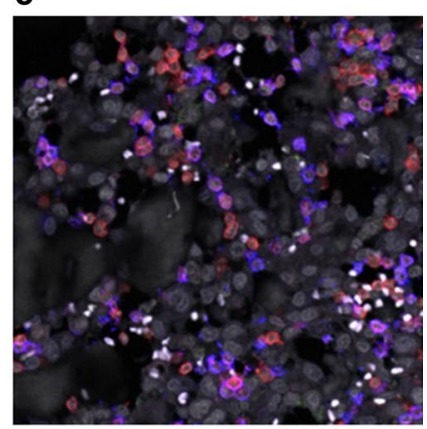

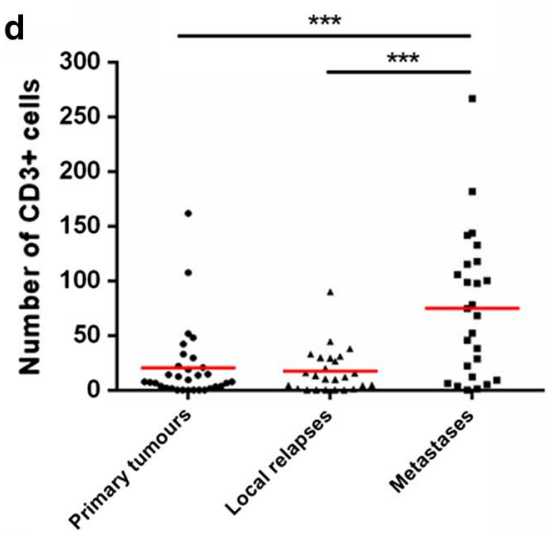

Fig. 2 Characterisation of T-cell density and subtypes in osteosarcoma. Representative images for tumour-infiltrating lymphocytes in primary tumour (a) and the associated local relapse (b) and metastasis (c). Triple immunofluorescent staining using anti-CD3 (red), anti-CD8 (blue) and anti-FOXP3 (green) was used to identify

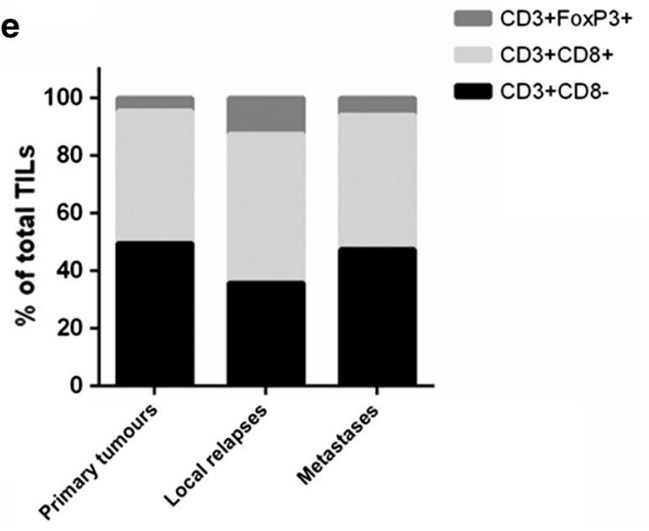

$\mathrm{CD}^{+}{ }^{+} \mathrm{CD} 8^{-}, \mathrm{CD}^{+}{ }^{+} \mathrm{CD} 8^{+}$and $\mathrm{CD} 3^{+} \mathrm{CD} 8^{-} \mathrm{FOXP} 3^{+} \mathrm{T}$ cells. Density of T-cell infiltration was higher in metastatic lesions (d), but the proportion of $\mathrm{CD}^{+}{ }^{+} \mathrm{CD} 8^{-}$and $\mathrm{CD} 3^{+} \mathrm{CD} 8^{+} \mathrm{T}$ cells was comparable with primary tumours $(\mathbf{e}) . \mathrm{CD}^{+} \mathrm{CD}^{-} \mathrm{FOXP} 3^{+} \mathrm{T}$ cells were found more frequently in local relapses 
the HLA-A expression status was added to the prediction model. Indeed, when we considered tumours with positive HLA class I expression including both HLA-A and HLA$\mathrm{B} / \mathrm{C}$, the mean number of $\mathrm{T}$ cells $\left(39 \pm 7 \mathrm{CD}^{+}\right.$cells) was not significantly higher compared to tumours with heterogeneous $\left(33 \pm 10 \mathrm{CD}^{+}\right.$cells; $\left.p=0.52\right)$ or negative HLA class I expression $\left(25 \pm 18 \mathrm{CD}^{+}\right.$cells; $\left.p=0.43\right)$. However, T-cell infiltrate was highly heterogeneous within the tumours and we noticed in tumours with heterogeneous HLA expression that tumour areas expressing more $\beta 2$-microglobulin and one of the HLA class I molecules contained more $\mathrm{T}$ cells than tumour areas with low expression of HLA class I. Consecutive cut sections stained for HLA class I and the triple CD3-CD8-FOXP3 combination were scanned using a Panoramic MIDI scanner in order to illustrate this observation of spatial colocalisation on selected areas, as shown in Supplementary Figure 4.

\section{High T-cell infiltrate associates with PD-L1 expression}

As PD-L1 expression is a possible mechanism for tumours to evade immune-mediated destruction, its expression was determined by immunohistochemistry on whole tumour sections. Membranous PD-L1 expression on $\geq 1 \%$ of osteosarcoma cells and/or immune cells (mainly macrophages) was found in 22 of 79 evaluated tumours (27.8\%). Representative images for PD-L1 staining on a progressive disease are shown in Fig. 3a-c. PD-L1 positivity was significantly higher in metastatic lesions (48\%) compared to local relapses and primary tumours (25 and $13 \%$, respectively; $p=0.004$ ) (Fig. 3d). High density of total $\mathrm{CD}^{+}$tumourinfiltrating $\mathrm{T}$ cells and $\mathrm{CD} 3^{+} \mathrm{CD} 8^{+} \mathrm{T}$ cells correlated with PD-L1 expression ( $p=0.002$ and $p=0.001$, respectively) (Fig. 3e, f). We also noticed a frequent spatial colocalisation between $\mathrm{T}$ cells and areas of the tumour with PD-L1 positivity. Six of the 17 patients with a known progressive disease stained positive for PD-L1 in metastases but not in primary tumours, and two patients had PD-L1 expression on both primary tumour and metastasis. PD-L1-positive metastastic lesions were significantly more infiltrated by $\mathrm{T}$ cells than PD-L1 negative lesions (mean $\pm \mathrm{SE}=123 \pm 20$ vs. $40 \pm 19 \mathrm{CD}^{+}$cells; $p=0.012$ ).

\section{Prognostic significance of immune markers in primary tumours and metastases}

Although mostly patients with poor outcome were included in this study, the time to progression differed between these a

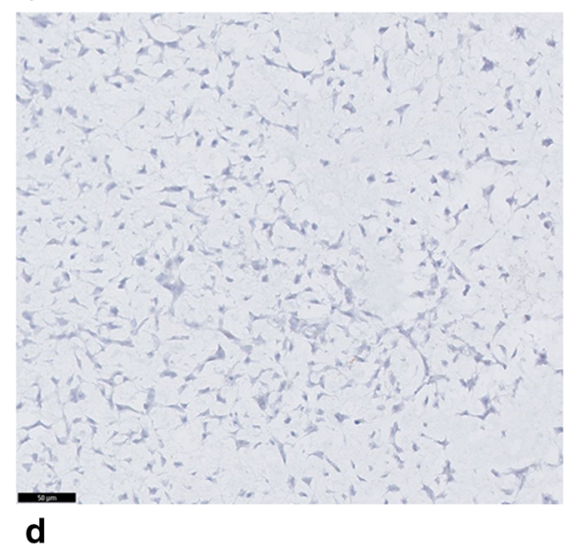

d

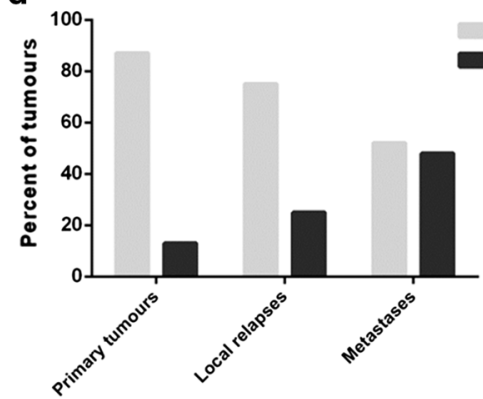

b

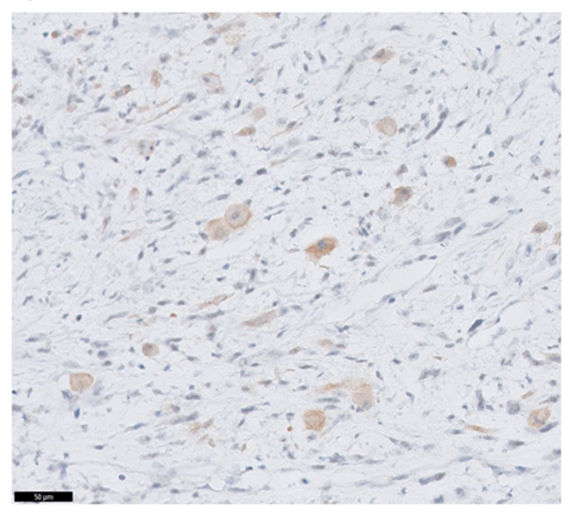

e

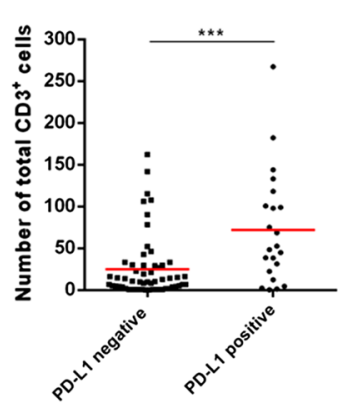

c

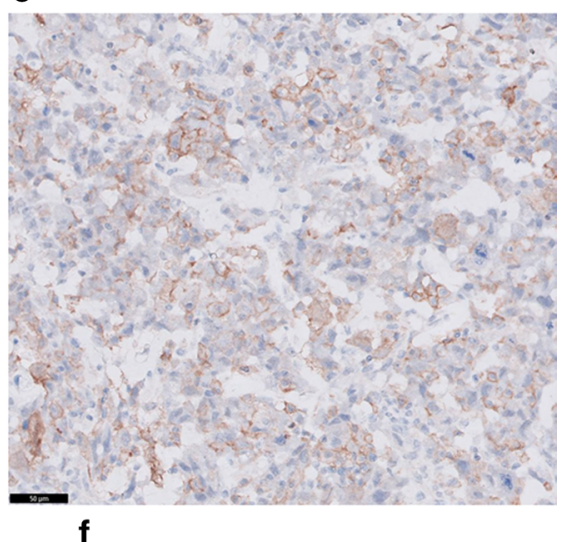

f

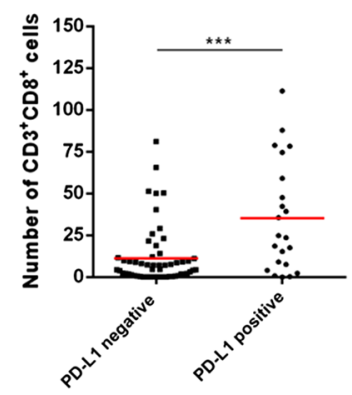

Fig. 3 High PD-L1 expression in metastatic osteosarcoma lesions. Representative images for PD-L1 immunostaining in an osteosarcoma patient. PD-L1 was negative in the primary tumour (a) while a membranous expression was detected on isolated cells (mainly macrophages) in the local relapse (b) and was more diffuse and observed on both osteosarcoma cells and immune cells in the lung metastasis (c). Scale bars $50 \mu \mathrm{m}$. PD-L1 expression was observed more frequently in metastatic osteosarcoma lesions, compared to primary tumours and local relapses (d). T-cell infiltration was higher in PD-L1 positive tumours $(\mathbf{e}, \mathbf{f})$ 

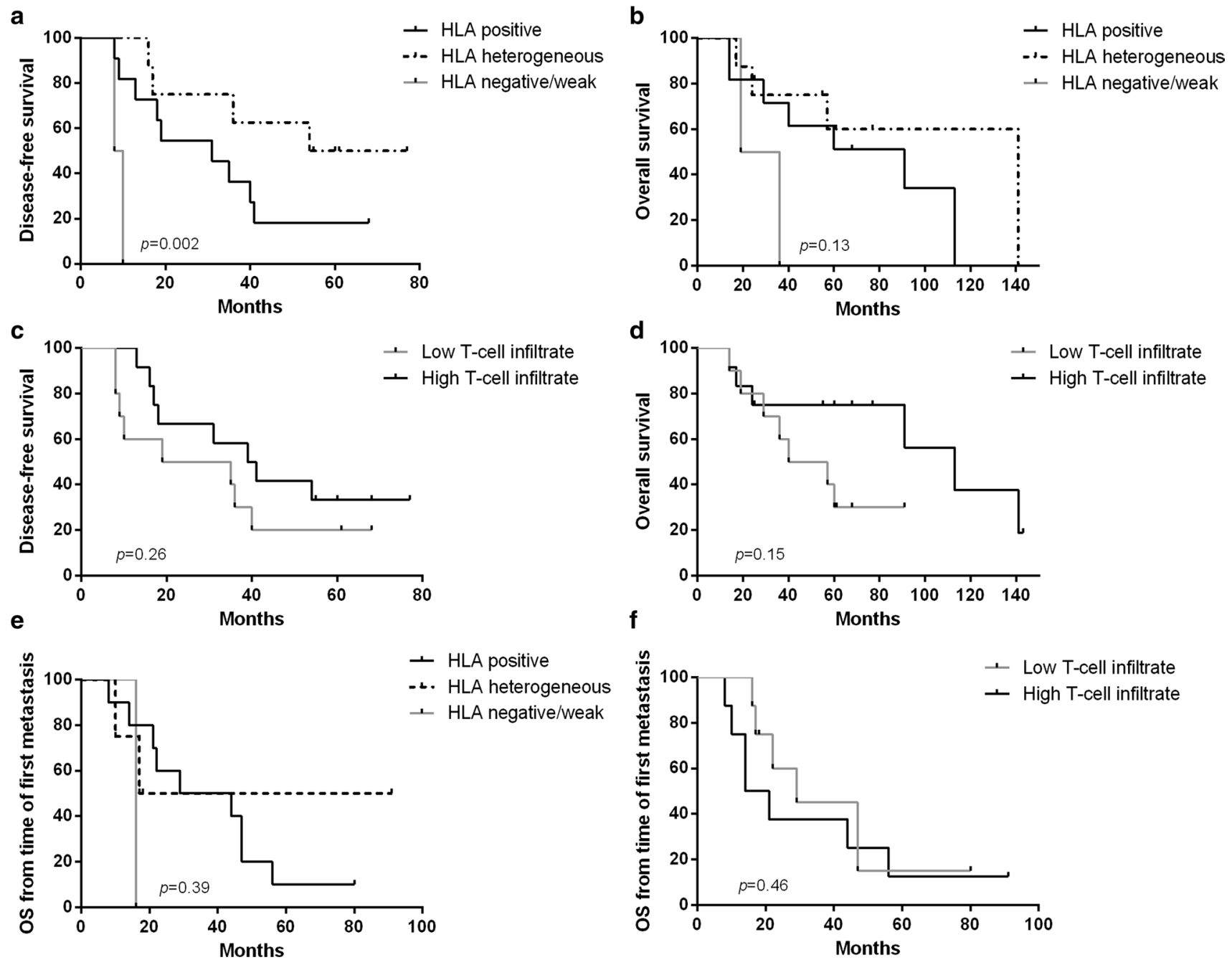

Fig. 4 Correlation between HLA class I status, total $\mathrm{T}$ cells and patient survival. Kaplan-Meier survival curves for disease-free survival and overall survival according to HLA class I status (a, b) and density of T-cell infiltration $(\mathbf{c}, \mathbf{d})$ of the primary tumour. Overall sur-

vival from the time of diagnosis of metastatic disease according to HLA class I status (e) and T-cell infiltration (f) of the first metastatic lesion. $p$ value obtained by log-rank test

patients, and there were also patients with non-progressive disease. Therefore, the prognostic significance of HLA class I expression, PD-L1 and T-cell infiltrate was assessed in the evaluable primary tumours $(n=22)$, as well as in the first metastatic lesion $(n=16)$, using Kaplan-Meier survival analysis. Heterogeneous and positive HLA class I expression in the primary tumour associated with a better disease-free survival compared to a negative/weak expression ( $p=0.001$ and $p=0.025$, respectively; overall comparison between the three groups $p=0.002$ ). However, a trend but no significant correlation was found for the overall survival $(p=0.13$ ) (Fig. $4 a, b)$. To evaluate the significance of infiltrating $\mathrm{T}$ cells, patients were divided in two groups based on the median numbers of total $\mathrm{CD}^{+}$cells (high and low). Patients with high T-cell infiltrate in the primary tumour tended to have a better clinical outcome although this was

not significant. For this group of patients, the median disease-free survival was 39 versus 19 months for patients with low T-cell infiltrate $(p=0.26)$ and the difference in the median overall survival was more pronounced (112 vs. 40 months; $p=0.15$ ) (Fig. $4 \mathrm{~d}$, e). Neither HLA class I expression nor density of T-cell infiltrate in the first metastatic lesion was related to overall survival from the time of diagnosis of the metastatic disease (Fig. 4c, f). PD-L1 status in either primary tumours or metastases did not correlate with patient survival (Supplementary Figure 5).

\section{Discussion}

In this study, we investigated HLA class I and PD-L1 expression, as well as T-cell infiltration in osteosarcoma, 
to assess the potential application of T-cell-based immunotherapies. To characterise the HLA class I expression, we used the monoclonal antibodies HCA2 and HC10, which recognise HLA-A and HLA-B/C heavy chains, respectively, and an antibody specific for the light chain $\beta 2$-microglobulin. Overall, we observed defects in HLA class I expression in $44 \%$ of the osteosarcomas, mainly with a heterogeneous pattern instead of a complete lack of HLA class I. Previous studies have reported a loss or a down-regulation of HLA class I expression in approximately $50-62 \%$ of osteosarcomas, using a pan-HLA class I antibody (EMR8-5) and a cut-off of 50\% positive tumour cells $[16,17]$. In our series, negative or heterogeneous expression of HLA-A molecules was found more frequently compared to the other heavy chains HLA-B/C and $\beta 2$-microglobulin. The clinical and therapeutic relevance of this finding, if any, has to be further investigated, since HLA-A and HLA-B both are antigen presenting molecules, and required for T-cell-based immunotherapies.

Another major clinical finding in our series is the high density of tumour-infiltrating $\mathrm{T}$ cells in metastatic osteosarcoma lesions compared to primary tumours and local relapses. $\mathrm{CD} 3^{+} \mathrm{CD} 8^{-}$and $\mathrm{CD} 3^{+} \mathrm{CD} 8^{+} \mathrm{T}$ cells were found in the same proportion within the tumour microenvironment while the density of $\mathrm{CD}^{+}{ }^{+} \mathrm{CD} 8^{-} \mathrm{FOXP}^{+}{ }^{+} \mathrm{T}$ cells was higher in local relapses, probably reflecting local immune escape mechanisms. Expression of the light chain $\beta 2$-microglobulin together with the HLA-B/C was an important predictor of T-cell infiltration. In other cancers, particularly with high mutational burden such as melanoma, lung and colorectal cancers, a high T-cell infiltrate commonly associates with better clinical outcome and may predict response to immunotherapy [10, 18, 19]. Although not statistically significant, high T-cell infiltrate in primary osteosarcomas also tended to have survival benefit for the patients included in our study. It has been demonstrated that tumour-infiltrating lymphocytes could be easily isolated from adult osteosarcomas and exhibited in vitro a high cytotoxic activity, suggesting that therapies based on these tumour-infiltrating $\mathrm{T}$ cells could be an efficient strategy in osteosarcoma [20]. However, some tumours are poorly infiltrated by $\mathrm{T}$ cells and the mechanisms associated with reduced T-cell trafficking and infiltration are poorly understood. A potential mechanism might be the loss of the tumour suppressor gene PTEN, frequently reported in osteosarcomas [21, 22].

PD-L1 positivity in metastases, while mainly negative in the associated primary tumours, emphasises the dynamics of an adaptive mechanism of immune escape. Overall, PD-L1 expression was found in almost half of the metastatic osteosarcoma lesions $(48 \%)$, on both osteosarcoma cells and immune cells, mainly macrophages. Our results are in accordance with other groups who reported
PD-L1 positivity using another monoclonal antibody only in metastatic osteosarcomas, as well as a higher PD-L1 mRNA expression in metastases, which correlated with the T-cell infiltrate [23, 24]. An increased PD-1 expression on peripheral $\mathrm{CD} 4^{+} \mathrm{T}$ cells was also observed in patients with metastases, strengthening the idea that PD-L1/PD-1 axis may play a role during osteosarcoma progression [25]. Recently, Koirala et al. reported the discrepancy between whole tumour sections and tissue micro-arrays as a result of the heterogeneity of PD-L1 expression in osteosarcoma, confirming our idea that this immune marker should be evaluated on whole sections [26]. Moreover, they identified PD-L1 positivity as a potential prognostic marker for poorer survival, which was not the case in our cohort. However, it should be noted that we mostly included patients with poor outcome, in order to assess the immune changes during disease progression. Therefore, our conclusions regarding the prognostic value of these different immune markers in primary tumours cannot be generalised in all osteosarcoma patients.

What is the explanation for the observed low and/or heterogeneous expression of HLA class I? In other cancers, defects in the antigen presentation pathway were reported at different levels (mutations in HLA-A, HLA-B and HLA$C$ genes, $\beta 2$-microglobulin or defects in components of antigen-processing machinery) and have been regarded as mechanisms to escape from T-cell immune recognition [27-29]. Tumour cells that have a loss/down-regulation of HLA class I may gain a selective clonal advantage in a process called immunoediting, enabling them to escape from the CD8+ T-cell-mediated destruction [30].

However, in osteosarcoma, the question can be raised regarding the mechanisms involved in the frequent heterogeneous expression of HLA class I. First of all, there is scarce data on baseline HLA class I expression in normal bone cells. Therefore, it is not clear whether low HLA class I expression reflects the normal situation or, in contrast, 'down regulation' as a consequence of escape from T-cell immune recognition. An alternative explanation for heterogeneity of HLA class I expression could be the consequence of the extreme genomic instability of osteosarcoma. But another interesting interpretation would be the induction of HLA class I expression by interferon- $\gamma$ secreted by $T$ cells. In this study, we observed that in tumours with HLA class I negative and positive regions, the T cells spatially colocalised with the HLA class I positive tumour areas. Additionally, we observed a strong correlation between high numbers of tumour-infiltrating $\mathrm{T}$ cells and PD-L1 expression. Together, this could imply that, in areas of T-cell infiltration, immune activation leads to induction of HLA class I and PD-L1 expression. Although it would have been interesting to look at the difference in immune profile of primary tumour between patients with and 
without metastasis at diagnosis, we could not answer the question due to the low number of samples.

Importantly, and despite the limited number of patients, HLA class I expression in primary osteosarcomas correlated with a better disease-free survival, which is consistent with previous results $[16,17]$. In addition, we observed that HLA class I expression in metastases was frequently positive, and this was in contrast to Tsukahara et al. [17] who reported that HLA class I loss or downregulation occurred more commonly in metastatic osteosarcoma lesions ( 7 out of 8 cases; $88 \%$ ). Consequently, the HLA data from our series can have two therapeutic implications. First, the frequent HLA class I expression in both primary tumours and metastases highlights the potential of (neo)antigen presentation by osteosarcoma cells, which can be exploitable in developing personalised immunotherapies. Second, identifying the rare patients with a negative or weak expression of HLA class I molecules on primary tumour is of clinical interest, to consider a different therapeutic approach (i.e. strategy based on NK cells) [31].

Altogether, the increased number of tumour-infiltrating $\mathrm{T}$ cells and PD-L1 expression during disease progression, associated with a frequent classical HLA class I expression, suggest that T-cell-based immunotherapy with adoptive cell transfer, peptide vaccines or immune checkpoint blockade could be a suitable treatment for metastatic osteosarcoma patients. Preclinical data demonstrated the benefit of PD-L1/PD-1 blockade antibodies, alone or in combination with anti-CTLA-4 in a mouse model of metastatic osteosarcoma and the efficacy of pembrolizumab, a monoclonal anti-PD-1 antibody, is currently being investigated for bone sarcomas in the phase II SARC028 study (NCT02301039) [23, 32]. Considering the limited therapeutic options currently available in advanced diseases, enhancing this preexisting antitumour immune response in metastastic lesions may offer clinical benefit in osteosarcoma patients.

\begin{abstract}
Acknowledgements We would like to thank Inge H. Briaire-de Bruijn and René Zwartbol for expert technical assistance in preparing the paraffin sections, Ekaterina S. Jordanova and Brendy E. van den Akker for help with the immunofluorescent procedures, as well as Noel F.C.C. de Miranda for providing the HLA antibodies and useful discussion. We also thank Ron Wolterbeek for his statistical assistance with the logistic regression model.
\end{abstract}

Funding Yayan T. Sundara was supported by the Indonesia Endowment Fund for Education (Lembaga Pengelola Dana Pendidikan/ LPDP), Ministry of Finance, Republic of Indonesia. Marie Kostine was supported in part by a grant from the Université de Bordeaux/ Centre Hospitalier Universitaire de Bordeaux, France. This work was performed in the context of EuroSARC, a collaborative project within the 7th Framework programme of the European Commission, under grant agreement 278742 .

\section{Compliance with ethical standards}

Conflict of interest The authors declare that they have no conflict of interest.

Open Access This article is distributed under the terms of the Creative Commons Attribution 4.0 International License (http://creativecommons.org/licenses/by/4.0/), which permits unrestricted use, distribution, and reproduction in any medium, provided you give appropriate credit to the original author(s) and the source, provide a link to the Creative Commons license, and indicate if changes were made.

\section{References}

1. Rosenberg A, Cleton-Jansen A, de Pinieux G (2013) Conventional osteosarcoma. In: Fletcher CDM, Bridge JA, Hogendoorn PC, Mertens F (eds) WHO classification of tumours of soft tissue and bone, 4th edn. IARC Press, Lyon, pp 282-288

2. The ESMO/European Sarcoma Network Working Group (2014) Bone sarcomas: ESMO clinical practice guidelines for diagnosis, treatment and follow-up. Ann Oncol 25(Suppl 3):113-123. doi:10.1093/annonc/mdu256

3. Kansara M, Teng MW, Smyth MJ, Thomas DM (2014) Translational biology of osteosarcoma. Nat Rev Cancer 14:722-735. doi:10.1038/nrc3838

4. Botter SM, Neri D, Fuchs B (2014) Recent advances in osteosarcoma. Curr Opin Pharmacol 16:15-23. doi:10.1016/j. coph.2014.02.002

5. Lorenz S, Barøy T, Sun J, Nome T, Vodák D, Bryne J-C et al (2016) Unscrambling the genomic chaos of osteosarcoma reveals extensive transcript fusion, recurrent rearrangements and frequent novel TP53 aberrations. Oncotarget 7:5273-5288. doi:10.18632/oncotarget.6567

6. Schumacher TN, Schreiber RD (2015) Neoantigens in cancer immunotherapy. Science 348:369-374. doi:10.1126/science. aaa4971

7. Lim J, Poulin NM, Nielsen TO (2015) New strategies in sarcoma: linking genomic and immunotherapy approaches to molecular subtype. Clin Cancer Res 21:4753-4759. doi:10.1158/10780432.CCR-15-0831

8. Coley WB II (1891) Contribution to the knowledge of sarcoma. Ann Surg 14:199-220

9. Cleton-Jansen A-M, Buddingh EP, Lankester AC (2012) Immunotherapy: is it different for sarcomas? Oncoimmunology 1:255257. doi:10.4161/onci.1.2.18345

10. Tumeh PC, Harview CL, Yearley JH, Shintaku IP, Taylor EJM, Robert L et al (2014) PD-1 blockade induces responses by inhibiting adaptive immune resistance. Nature 515:568-571. doi:10.1038/nature13954

11. Thor Straten P, Garrido F (2016) Targetless T cells in cancer immunotherapy. J Immunother Cancer. 4:23. doi:10.1186/ s40425-016-0127-z

12. Pardoll DM (2012) The blockade of immune checkpoints in cancer immunotherapy. Nat Rev Cancer 12:252-264. doi:10.1038/ $\operatorname{nrc} 3239$

13. Kostine M, Cleven AH, de Miranda NFCC, Italiano A, CletonJansen A-M, Bovée JVMG (2016) Analysis of PD-L1, T-cell infiltrate and HLA expression in chondrosarcoma indicates potential for response to immunotherapy specifically 
in the dedifferentiated subtype. Mod Pathol. doi:10.1038/ modpathol.2016.108

14. Garon EB, Rizvi NA, Hui R, Leighl N, Balmanoukian AS, Eder JP et al (2015) Pembrolizumab for the treatment of non-smallcell lung cancer. N Engl J Med 372:2018-2028. doi:10.1056/ NEJMoa1501824

15. Punt S, van Vliet ME, Spaans VM, de Kroon CD, Fleuren GJ, Gorter A et al (2015) FoxP3(+) and IL-17(+) cells are correlated with improved prognosis in cervical adenocarcinoma. Cancer Immunol Immunother 64:745-753. doi:10.1007/ s00262-015-1678-4

16. Nada OH, Ahmed NS, Abou Gabal HH (2014) Prognostic significance of HLA EMR8-5 immunohistochemically analyzed expression in osteosarcoma. Diagn Pathol. 9:72. doi:10.1186/1746-1596-9-72

17. Tsukahara T, Kawaguchi S, Torigoe T, Asanuma H, Nakazawa E, Shimozawa K et al (2006) Prognostic significance of HLA class I expression in osteosarcoma defined by anti-pan HLA class I monoclonal antibody, EMR8-5. Cancer Sci 97:1374-1380. doi:10.1111/j.1349-7006.2006.00317.x

18. Al-Batran S-E, Rafiyan M-R, Atmaca A, Neumann A, Karbach $\mathrm{J}$, Bender A et al (2005) Intratumoral T-cell infiltrates and MHC class I expression in patients with stage IV melanoma. Cancer Res 65:3937-3941. doi:10.1158/0008-5472.CAN-04-4621

19. Turcotte S, Katz SC, Shia J, Jarnagin WR, Kingham TP, Allen PJ et al (2014) Tumor MHC class I expression improves the prognostic value of T-cell density in resected colorectal liver metastases. Cancer Immunol Res. 2:530-537. doi:10.1158/2326-6066. CIR-13-0180

20. Théoleyre S, Mori K, Cherrier B, Passuti N, Gouin F, Rédini $F$ et al (2005) Phenotypic and functional analysis of lymphocytes infiltrating osteolytic tumors: use as a possible therapeutic approach of osteosarcoma. BMC Cancer 5:123. doi:10.1186/1471-2407-5-123

21. Peng W, Chen JQ, Liu C, Malu S, Creasy C, Tetzlaff MT et al (2016) Loss of PTEN promotes resistance to T Cell-mediated immunotherapy. Cancer Discov. 6:202-216. doi:10.1158/21598290.CD-15-0283

22. Moriarity BS, Otto GM, Rahrmann EP, Rathe SK, Wolf NK, Weg MT et al (2015) A sleeping beauty forward genetic screen identifies new genes and pathways driving osteosarcoma development and metastasis. Nat Genet 47:615-624. doi:10.1038/ ng.3293
23. Lussier DM, O’Neill L, Nieves LM, McAfee MS, Holechek SA, Collins AW et al (2015) Enhanced T-cell immunity to osteosarcoma through antibody blockade of PD-1/PD-L1 interactions. J Immunother 38:96-106. doi:10.1097/CJI.0000000000000065

24. Shen JK, Cote GM, Choy E, Yang P, Harmon D, Schwab J et al (2014) Programmed cell death ligand 1 expression in osteosarcoma. Cancer Immunol Res. 2:690-698. doi:10.1158/23266066.CIR-13-0224

25. Zheng W, Xiao H, Liu H, Zhou Y (2015) Expression of programmed death 1 is correlated with progression of osteosarcoma. APMIS 123:102-107. doi:10.1111/apm.12311

26. Koirala P, Roth ME, Gill J, Piperdi S, Chinai JM, Geller DS et al (2016) Immune infiltration and PD-L1 expression in the tumor microenvironment are prognostic in osteosarcoma. Sci Rep 6:30093. doi:10.1038/srep30093

27. Seliger B, Cabrera T, Garrido F, Ferrone S (2002) HLA class I antigen abnormalities and immune escape by malignant cells. Semin Cancer Biol 1:3-13. doi:10.1111/j.1349-7006.2006.00317.x

28. Seliger B (2008) Molecular mechanisms of MHC class I abnormalities and APM components in human tumors. Cancer Immunol Immunother 11:1719-1726. doi:10.1007/s00262-008-0515-4

29. Dierssen JW, de Miranda NF, Ferrone S, van Puijenbroek M, Cornelisse CJ, Fleuren GJ et al (2007) HNPCC versus sporadic microsatellite-unstable colon cancers follow different routes toward loss of HLA class I expression. BMC Cancer 22:7-33. doi:10.1186/1471-2407-7-33

30. Schreiber RD, Old LJ, Smyth MJ (2011) Cancer immunoediting: integrating immunity's roles in cancer suppression and promotion. Science 331:1565-1570. doi:10.1126/science.1203486

31. Fernández L, Valentín J, Zalacain M, Leung W, Patiño-Garcia A, Pérez-Martínez A (2015) Activated and expanded natural killer cells target osteosarcoma tumor initiating cells in an NKG2D-NKG2DL dependent manner. Cancer Lett 1:54-63. doi:10.1016/j.canlet.2015.07.042

32. Lussier DM, Johnson JL, Hingorani P, Blattman JN (2015) Combination immunotherapy with $\alpha$-CTLA-4 and $\alpha$-PD-L1 antibody blockade prevents immune escape and leads to complete control of metastatic osteosarcoma. J Immunother Cancer 3:21. doi:10.1186/s40425-015-0067-z 